\title{
新型含 $N$-吡啶基吡唑结构的吡唑肜类衍生物的合成与生物活性
}

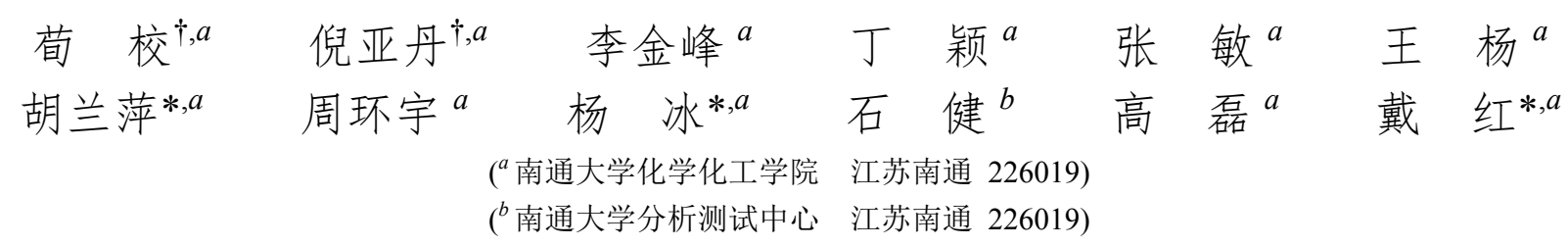

\begin{abstract}
摘要 为了探索与发现具有优良生物活性的吡唑肜类衍生物, 利用活性基团拼接法, 以杀螨剂唑螨酯为先导化合物, 引入 $N$-吡啶基吡唑结构单元, 设计合成了 20 个未见文献报道的新型吡唑肜类化合物, 化合物结构均经 ${ }^{1} \mathrm{H} N \mathrm{NMR},{ }^{13} \mathrm{C}$ NMR 和元素分析确认. 生物活性初步测试结果表明, 所有目标化合物在 $500 \mu \mathrm{g} / \mathrm{mL}$ 浓度下对东方粘虫均呈现出 $100 \%$ 的杀虫活性. 5 个化合物在 $100 \mu \mathrm{g} / \mathrm{mL}$ 下对东方粘虫的杀灭活性均达 $100 \%, 2$ 个化合物在 $20 \mu \mathrm{g} / \mathrm{mL}$ 下对东方粘虫仍具有 $40 \%$ 的杀虫效果. 此外, 3 个化合物在 $500 \mu \mathrm{g} / \mathrm{mL}$ 浓度下对苜葆蜴的致死率为 $40 \% \sim 60 \%$. 值得注意的是, 5 - $(2$-澳苯氧 基)-1,3-二甲基- $1 H$-吡唑-4-甲醛- $O$ - $\{1$-(3-氯吡啶-2-基)-3-[(6-氯吡啶-3-基)甲氧基]- $1 H$-吡唑-5-甲酰基 $\}$ 肜(10b)和 5-(4-叔 丁基苯氧基)-1,3-二甲基- $1 H$-吡唑-4-甲醛- $O$-\{1-(3-氯吡啶-2-基)-3-[(6-氯吡啶-3-基)甲氧基]- $1 H$-吡唑-5-甲酰基\}肜(10k)对 东方粘虫的杀虫活性较为突出, 可作深入的结构优化与杀虫活性探究.
\end{abstract}

关键词 $N$-吡啶基吡唑; 吡唑肜; 合成; 生物活性

\section{Synthesis and Bioactivities of Novel Pyrazole Oxime Derivatives Containing a N-Pyridylpyrazole Moiety}

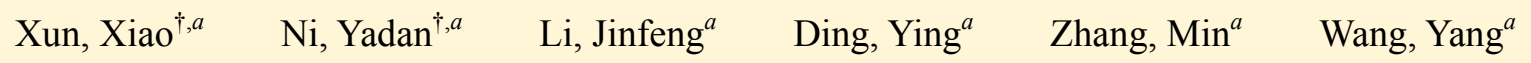 \\ Hu, Lanping ${ }^{*, a} \quad$ Zhou, Huanyu $^{a} \quad$ Yang, Bing ${ }^{*, a} \quad$ Shi, Jian ${ }^{b} \quad$ Gao, Lei ${ }^{a} \quad$ Dai, Hong ${ }^{*, a}$ \\ ( ${ }^{a}$ College of Chemistry and Chemical Engineering, Nantong University, Nantong, Jiangsu 226019) \\ ( ${ }^{b}$ Analysis and Testing Center, Nantong University, Nantong, Jiangsu 226019)
}

\begin{abstract}
In search of novel pyrazole oxime compounds with potent bioactivities, twenty pyrazole oxime derivatives were synthesized by introducing $N$-pyridylpyrazole unit into pyrazole oxime skeleton, based on the lead of fenpyroximate. The title compounds were structurually characterized by ${ }^{1} \mathrm{H}$ NMR, ${ }^{13} \mathrm{C}$ NMR and elemental analysis. The preliminary bioassay showed that all the title compounds had 100\% insecticidal activities against Mythimna separata Walker at $500 \mu \mathrm{g} / \mathrm{mL}$. Five compounds displayed $100 \%$ mortality rate towards Mythimna separata Walker at $100 \mu \mathrm{g} / \mathrm{mL}$. Two compounds both exhibited $40 \%$ mortality rate against Mythimna separata Walker at $20 \mu \mathrm{g} / \mathrm{mL}$. In addition, three compounds had $40 \sim 60 \%$ insecticidal activities to Aphis medicaginis at $500 \mu \mathrm{g} / \mathrm{mL}$. It is worthy of noting that 5-(2-bromophenoxy)-1,3-dimethyl- $1 H$-pyrazole-4-carbaldehyde$O$ - $\{1$-(3-chloropyridin-2-yl)-3-[(6-chloropyridin-3-yl)methoxy]- $1 H$-pyrazole-5-formyl $\}$ oxime $\quad(10 b)$ and 5 -(4- $t$-butylphenoxy)-1,3-dimethyl-1 $H$-pyrazole-4-carbaldehyde- $O$ - $\{1$-(3-chloropyridin-2-yl)-3-[(6-chloropyridin-3-yl)methoxy]-1H-pyrazole5-formyl \}oxime (10k) which have potent insecticidal activities against Mythimna separata Walker, can be utilized in insecticide research with further optimization.
\end{abstract}

Keywords $N$-pyridylpyrazole; pyrazole oxime; synthesis; bioactivity

目前，氮杂环类化合物因具有多样化的结构形式、 广谱的生物活性而成为新药创制的热点领域 ${ }^{[1 \sim 11]} . N$-吡
啶基吡唑类化合物是一种重要的含氮杂环, 在农药领域

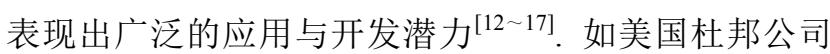

\footnotetext{
* Corresponding authors. E-mail: hlp@ntu.edu.cn; yangbing111@ntu.edu.cn; daihong_2015@aliyun.com

Received February 18, 2020; revised March 6, 2020; published online March 11, 2020.

Project supported by the National Natural Science Foundation of China (No. 21372135) and the Science and Technology Project Fund of Nantong City (No. MS12019060).

国家自然科学基金(No. 21372135)和南通市科技计划(No. MS12019060)资助项目.

共同第一作者(These authors contributed equally to this work).
} 
开发并商品化的杀虫剂氯虫苯甲酰胺(Chlorantraniliprole, 图 1)和溴氰虫酰胺(Cyantraniliprole, 图 1)对小菜蛾、 东方粘虫、斜纹夜蛾、苜宿蚜及飞虫等害虫显示出优异 的防治效果, 该类化合物均含有 $N$-吡啶基吡唑活性单 元，具有高效、低毒、与传统农药无交互抗性等特点 ${ }^{[18]}$. 由于该类杀虫剂的发现, 有关其 $N$-吡啶基吡唑类衍生 物的分子设计、合成与生物活性研究迅速成为农药化学 领域的热点课题之一 ${ }^{[19 ~ 22]}$. 吡唑肜类化合物作为含氮 杂环家族中重要一员, 亦具有良好生物活性如杀菌、杀 虫、抗癌和抗病毒等, 常常被应用于农业生产与医疗保 健等方面 ${ }^{[23 ~ 26]}$. 近年来已商品化的代表性化合物有日 本 Nihon Nohyaku 公司研制的杀螨剂唑螨酯 (Fenpyroximate, 图 1), 该化合物对红蜘蛛、附线螨及瘘 螨等害虫展现优良的杀灭作用, 具有受季节影响较小、 持效时间较长等优点 ${ }^{[27]}$, 多年来唑螨酯一直作为农用 杀螨剂在农作物保护领域发挥着举足轻重的作用. 然而 随着唑螨酯的广泛应用, 其抗药性问题也愈发凸显出 来 ${ }^{[28]}$. 因此, 为发现新的活性物质, 农药工作者纷纷以 唑螨酯分子结构中吡唑肜骨架为中心, 引入不同杂环活
性基团, 研发出了不少具有杀虫活性的吡唑肜衍生物, 如 $\mathrm{Fu}$ 等 ${ }^{[29]}$ 在唑螨酯结构中引入吡啶杂环, 合成出的含 吡啶单元的吡唑肜化合物 $\mathbf{A}$ (图 1)显示优异杀虫活性, 在 $100 \mu \mathrm{g} / \mathrm{mL}$ 浓度下对螨虫致死率达 $100 \%$, Yang 等 ${ }^{[30]}$ 在唑螨酯结构上引入噻唑杂环, 研究发现含噻唑环结构 的吡唑肜化合物 $\mathbf{B}$ (图 1)具有较好的杀虫作用, 在 5 $\mathrm{mg} / \mathrm{L}$ 浓度下对蚊虫杀灭活性达 $100 \%$, 在 $200 \mathrm{mg} / \mathrm{L}$ 浓度 下对螨虫致死率为 $95 \%$. 本课题组近年来在唑螨酯结构 上引入呋喃杂环, 衍生合成出的吡唑肜化合物 $\mathbf{C}$ (图 1) 也表现出较好杀虫活性 ${ }^{[31]}$, 在 $400 \mu \mathrm{g} / \mathrm{mL}$ 浓度下对螨虫 具有 $83 \%$ 的防效. 为了进一步从吡唑肟类化合物中开发 出具有良好生物活性的物质, 本研究在前期工作的基础 上, 以杀螨剂唑螨酯为农药先导化合物, 采用活性基团 拼接原理, 用 $N$-吡啶基吡唑活性单元替代唑螨酯结构 中的取代苯环部分, 设计合成了一系列含 $N$-吡啶基吡 唑结构的吡唑肜类衍生物(图 2), 同时篮选其对螨虫、东 方粘虫和苜宿蚜的杀虫活性. 具体合成路线如 Scheme 1 所示.<smiles>CNC(=O)c1cc(Cl)cc(C)c1NC(=O)c1cc(Br)nn1-c1ncccc1Cl</smiles><smiles>Cc1nn(C)c(Oc2cccc(Br)c2)c1/C=N/OCc1ccc(C(=O)OC(C)(C)C)cn1</smiles><smiles>CNC(=O)c1cc(C#N)cc(C)c1NC(=O)c1cc(Br)nn1-c1ncccc1Cl</smiles><smiles>Cc1nn(C)c(Oc2ccccc2Cl)c1/C=N/OCc1cnc(Cl)s1</smiles><smiles>Cc1nn(C)c(Oc2ccccc2)c1/C=N\OCc1ccc(C(=O)OC(C)(C)C)cc1</smiles><smiles>Cc1nn(C)c(Oc2ccc(Cl)cc2Cl)c1/C=N/OC(=O)c1ccc(-c2ccc(C(F)(F)F)cc2)o1</smiles>

图 1 氯虫苯甲酰胺、溴氰虫酰胺、唑螨酯和化合物 $\mathbf{A} \sim \mathbf{C}$ 的化学结构

Figure 1 Chemical structures of chlorantraniliprole, cyantraniliprole, fenpyroximate and compounds $\mathbf{A} \sim \mathbf{C}$
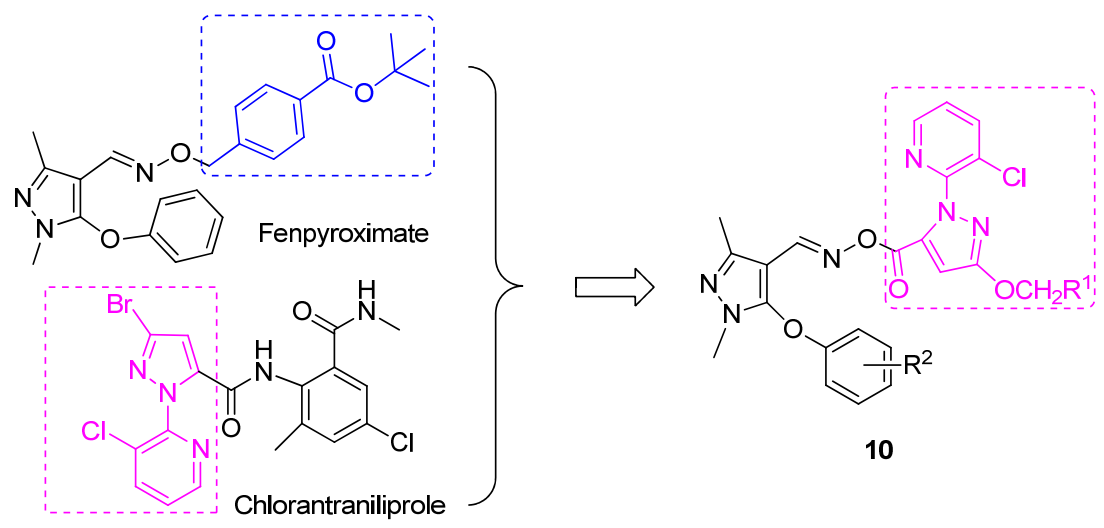

10

图 2 目标化合物 $\mathbf{1 0}$ 的分子设计

Figure 2 Design of the target compound 10 

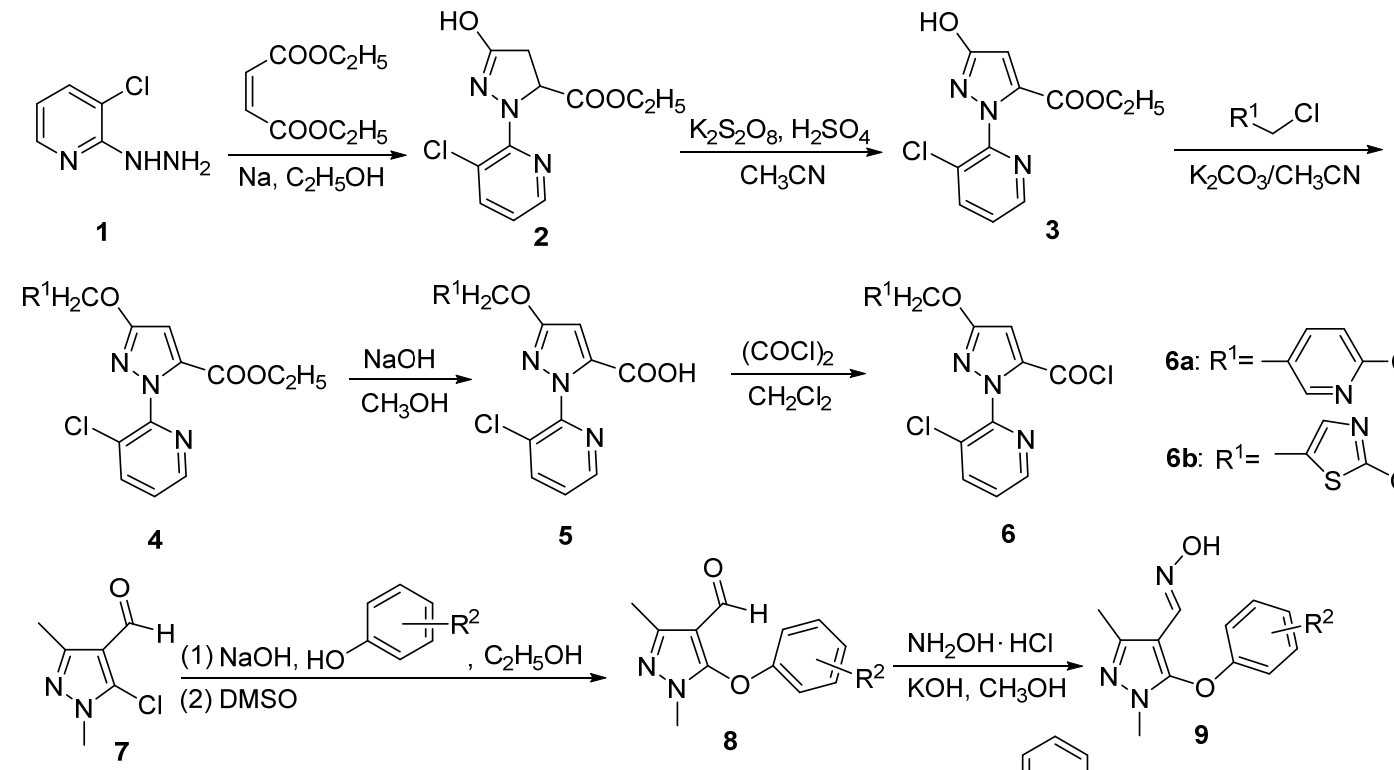

$\mathrm{R}^{1} \mathrm{H}_{2} \mathrm{CO}$

$\mathrm{R}^{1} \mathrm{H}_{2} \mathrm{CO}$<smiles>[R]COc1cc(C(=O)Cl)n(-c2ncccc2Cl)n1</smiles><smiles>Cc1nn(C)c(OC=C2[R]CCCC2)c1/C=N/O</smiles><smiles>Cc1nn(C)c(Oc2cccc(F)c2)c1C=O</smiles>

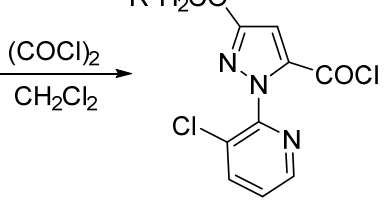<smiles>[R19]=[R19]c1ccc(Cl)nc1</smiles>

6

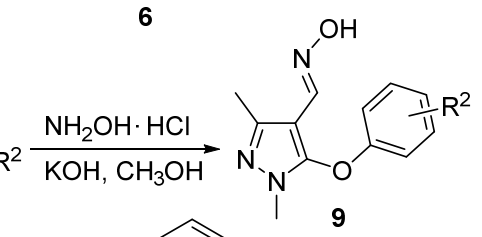<smiles>[R]COc1cc(C(=O)O/N=C/c2c(C)nn(C)c2Oc2ccccc2)n(-c2ncccc2Cl)n1</smiles>

$10 \mathrm{a} \sim 10 \mathrm{t}$

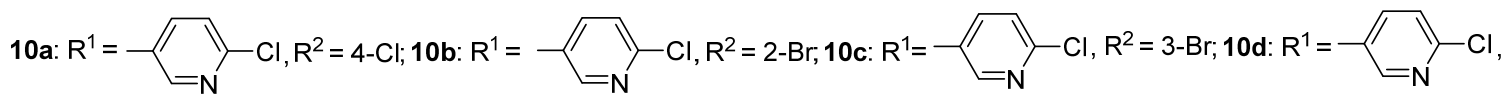
$\mathrm{R}^{2}=\mathrm{H} ; 10 \mathrm{e}: \mathrm{R}^{1}=\mathrm{N}_{\mathrm{N}} \mathrm{Cl}, \mathrm{R}^{2}=2-\mathrm{OCH}_{3} ; 10 \mathrm{f}: \mathrm{R}^{1}=\mathrm{N}_{\mathrm{N}} \mathrm{Cl}, \mathrm{R}^{2}=4-\mathrm{OCH}_{3} ; \mathbf{1 0 g}: \mathrm{R}^{1}=2-\mathrm{RCF}_{3}$; 10h: $\mathrm{R}^{1}=-\mathrm{Cl}, \mathrm{R}^{2}=4-\mathrm{OCF}_{3} ; 10 \mathrm{i}: \mathrm{R}^{1}=\mathrm{N}_{\mathrm{N}} \mathrm{Cl}, \mathrm{R}^{2}=3-\mathrm{CH}_{3} ; 10 \mathrm{j}: \mathrm{R}^{1}=4-\mathrm{CH}_{3}$;

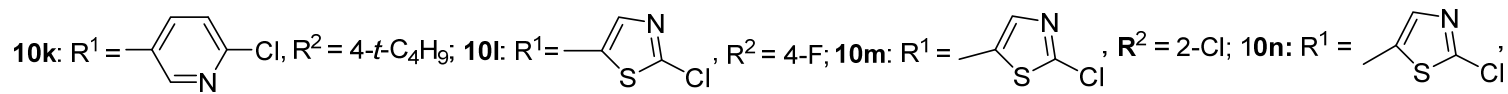

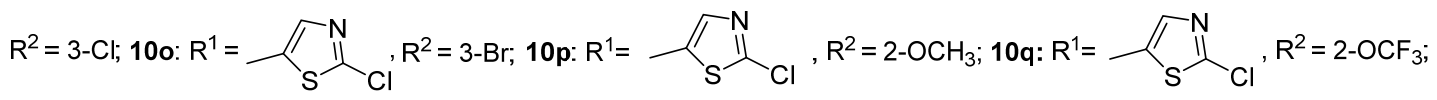
10r: $R^{1}=\overbrace{S}^{N}, R_{C l}^{2}=4-C_{3} ; 10 s: R^{1}=\overbrace{S}^{N}\|_{C l}^{N}, R^{2}=4-t-C_{4} H_{9} ; 10 t: R^{1}=\overbrace{S}^{N}\|_{C l}^{N}, R^{2}=2,3-\left(C H_{3}\right)_{2}$

图式 1 目标化合物 $\mathbf{1 0}$ 的合成路线

Scheme 1 Synthetic route of the title compound 10

\section{1 结果与讨论}

\section{1 目标产物 10 的合成}

在目标化合物 10 的制备过程中, 选取化合物 $10 \mathrm{j}$ 为 例, 对比了不同的溶剂、缚酸剂对化合物 $10 \mathbf{j}$ 收率的影 响(表 1). 研究发现, 当选用三乙胺作为缚酸剂, 以二氯 甲烷或四氢呋喃作反应溶剂时, 室温摚拌 $12 \mathrm{~h}$, 收率分 别为 $56 \%$ 和 $53 \%$, 薄层色谱(TLC)检测显示, 仍有部分 原料未发生反应, 换为极性较大的乙腈作为反应溶剂 时, 室温搅拌 $12 \mathrm{~h}$, 收率为 $65 \%$, 比二氯甲烷或四氢呋
喃作溶剂时收率有了一定的提高; 以三乙胺作为缚酸 剂, 以二氯甲烷或乙腈作溶剂, 将室温摚拌改为加热回 流 $12 \mathrm{~h}$, 化合物 $\mathbf{1 0 j}$ 的收率分别为 $50 \%$ 和 $61 \%$, 略低于 室温搅拌的结果. 选用吡啶作为缚酸剂, 分别以二氯甲 烷、四氢呋喃和乙腈作溶剂, 室温搅拌 $12 \mathrm{~h}$, 收率分别 为 $57 \%$ 、35\% 和 $60 \%$, 比三乙胺作缚酸剂时收率没有得 到明显改善; 以吡啶作为缚酸剂, 二氯甲烷或乙腈作溶 剂, 室温搅拌改为加热回流 $12 \mathrm{~h}$, 化合物 $\mathbf{1 0 j}$ 的收率分 别为 $52 \%$ 和 $54 \%$, 收率略有降低. 随后以乙腈作为溶剂, 分别以碳酸钠、碳酸钾和氢氧化钾作为缚酸剂, 室温摚 
拌 $12 \mathrm{~h}$, 发现反应体系容易结块, TLC 检测显示仍有大 部分原料未发生反应, 后续分离比较困难, 其收率均较 低, 分别为 $12 \% 、 10 \%$ 和 $15 \%$. 由此可见, 选用三乙胺作 缚酸剂, 乙腈作溶剂, 室温摚拌 $12 \mathrm{~h}$ 是合成目标产物的 最佳方法，同时后续处理也较为简便. 因此，通过该方 法顺利合成出了 20 个含 $N$-吡啶基吡唑单元的新型吡唑 肜类化合物.

\section{表 1 不同反应条件对目标物 $10 \mathrm{j}$ 收率的影响}

Table 1 Effect of different reaction conditions on the yield of target compound $\mathbf{1 0 j}$

\begin{tabular}{|c|c|c|c|c|}
\hline Entry & Base & Solvent & Reaction condition & Yield/\% \\
\hline 1 & $\mathrm{Et}_{3} \mathrm{~N}$ & $\mathrm{CH}_{2} \mathrm{Cl}_{2}$ & Room temperature for $12 \mathrm{~h}$ & 56 \\
\hline 2 & $\mathrm{Et}_{3} \mathrm{~N}$ & THF & Room temperature for $12 \mathrm{~h}$ & 53 \\
\hline 3 & $\mathrm{Et}_{3} \mathrm{~N}$ & $\mathrm{CH}_{3} \mathrm{CN}$ & Room temperature for $12 \mathrm{~h}$ & 65 \\
\hline 4 & $\mathrm{Et}_{3} \mathrm{~N}$ & $\mathrm{CH}_{2} \mathrm{Cl}_{2}$ & Reflux for $12 \mathrm{~h}$ & 50 \\
\hline 5 & $\mathrm{Et}_{3} \mathrm{~N}$ & $\mathrm{CH}_{3} \mathrm{CN}$ & Reflux for $12 \mathrm{~h}$ & 61 \\
\hline 6 & Pyridine & $\mathrm{CH}_{2} \mathrm{Cl}_{2}$ & Room temperature for $12 \mathrm{~h}$ & 57 \\
\hline 7 & Pyridine & THF & Room temperature for $12 \mathrm{~h}$ & 35 \\
\hline 8 & Pyridine & $\mathrm{CH}_{3} \mathrm{CN}$ & Room temperature for $12 \mathrm{~h}$ & 60 \\
\hline 9 & Pyridine & $\mathrm{CH}_{2} \mathrm{Cl}_{2}$ & Reflux for $12 \mathrm{~h}$ & 52 \\
\hline 10 & Pyridine & $\mathrm{CH}_{3} \mathrm{CN}$ & Reflux for $12 \mathrm{~h}$ & 54 \\
\hline 11 & $\mathrm{Na}_{2} \mathrm{CO}_{3}$ & $\mathrm{CH}_{3} \mathrm{CN}$ & Room temperature for $12 \mathrm{~h}$ & 12 \\
\hline 12 & $\mathrm{~K}_{2} \mathrm{CO}_{3}$ & $\mathrm{CH}_{3} \mathrm{CN}$ & Room temperature for $12 \mathrm{~h}$ & 10 \\
\hline 13 & $\mathrm{KOH}$ & $\mathrm{CH}_{3} \mathrm{CN}$ & Room temperature for $12 \mathrm{~h}$ & 15 \\
\hline
\end{tabular}

\section{2 目标产物 10 的核磁数据解析}

以目标物 10c 为例, 对其 ${ }^{1} \mathrm{H} N M R$ 与 ${ }^{13} \mathrm{C} N M R$ 数据 进行分析. 从 10c 的 ${ }^{1} \mathrm{H}$ NMR 谱图可以看出, $\mathrm{CH}=\mathrm{N}$ 中 的 $\mathrm{H}$ 受 $\mathrm{N}$ 原子诱导作用的影响向低场移动, 在 $\delta 7.92$ 以 单峰出现; $3-\mathrm{Br}$ 取代苯环上的 1 个 $\mathrm{H}$ 在 $\delta 7.31$ 以双重峰 出现, 其余 3 个 $\mathrm{H}$ 分别在 $\delta 7.09 \sim 7.25$ 和 $6.82 \sim 6.85$ 以 多重峰出现; 吡唑环上 4-位氢在 $\delta 6.48$ 以单峰出现; 与 吡啶环相连的 $\mathrm{OCH}_{2}$ 中的 2 个 $\mathrm{H}$ 由于受到 $\mathrm{O}$ 原子和吡啶 环诱导作用的影响向低场移动, 在 $\delta 5.31$ 以单峰出现; 吡唑环 1-位甲基上 3 个 $\mathrm{H}$ 由于受 $\mathrm{N}$ 原子诱导作用的影 响向低场移动, 在 $\delta 3.60$ 以单峰形式出现; 吡唑环 3-位 甲基上 3 个 $\mathrm{H}$ 在 $\delta 2.44$ 以单峰出现. 从化合物 $10 \mathrm{c}$ 的 ${ }^{13} \mathrm{C}$ NMR 数据可知, 酯基上羰基 $\mathrm{C}$ 原子的 $\delta$ 值为 162.5 ; $\mathrm{CH}=\mathrm{N}$ 上的 $\mathrm{C}$ 原子受 $\mathrm{N}$ 原子诱导作用的影响向低场移 动, $\delta$ 值为 151.3 ; 与吡啶环相连的 $\mathrm{OCH}_{2}$ 上的 $\mathrm{C}$ 原子, 由 于相邻 $\mathrm{O}$ 原子和吡啶环的诱导作用向低场移动, $\delta$ 值为 67.6; 吡坐环 3 位甲基上的 $\mathrm{C}$ 原子受 $\mathrm{N}$ 原子诱导作用的 影响向低场移动, $\delta$ 值为 34.5 ; 吡唑环 3 位甲基上的 $\mathrm{C}$ 原 子的 $\delta$ 值为 15.3 .

\section{3 生物活性}

目标产物 $10 \mathrm{a} \sim 10 \mathrm{t}$ 对东方粘虫 (Mythimna separata Walker)、苜葆蚜(Aphis medicaginis)和朱砂叶螨(Tetranychus cinnabarinus) 的杀虫活性数据列于表 2 . 表 2 显示,
在 $500 \mu \mathrm{g} / \mathrm{mL}$ 测试浓度下，化合物 10a 10t 对东方粘虫 均表现出优异的杀虫作用，其杀虫活性均为 $100 \%$, 与 对照药剂唑虫酰胺的防效相当. 当 $\mathrm{R}^{1}$ 为 6-氯吡啶-3-基 时, 化合物 10b $\left(R^{2}=2-B r\right) 、 10 d\left(R^{2}=H\right)$ 和 10k $\left(R^{2}=\right.$ $\left.4-t-\mathrm{C}_{4} \mathrm{H}_{9}\right)$ 在 $100 \mu \mathrm{g} / \mathrm{mL}$ 浓度下对东方粘虫的致死率均达 $100 \%$, 对东方粘虫杀虫活性高. 从构效关系可以看出, 当苯环上引入氯、溴吸电子基团时, 其取代基位置对杀 虫活性有一定的影响, 邻位取代物对东方粘虫的杀虫活 性优于间位或对位的杀虫效果, 如化合物 $10 b\left(R^{2}=\right.$ 2-Br) 对东方粘虫的杀虫活性为 $100 \%$, 明显高于 $10 \mathrm{c}$ $\left(\mathrm{R}^{2}=3-\mathrm{Br}\right)$ 和 10a $\left(\mathrm{R}^{2}=4-\mathrm{Cl}\right)$ 的防效, 当苯环对位引入甲 氧基、三氟甲氧基、甲基、叔丁基给电子基团时，化合 物 10k $\left(\mathrm{R}^{2}=4-t-\mathrm{C}_{4} \mathrm{H}_{9}\right)$ 对东方粘虫的杀虫活性要优于化 合物 $10 f\left(\mathrm{R}^{2}=4-\mathrm{OCH}_{3}\right) 、 10 \mathrm{~h}\left(\mathrm{R}^{2}=4-\mathrm{OCF}_{3}\right)$ 和 $10 \mathrm{j}\left(\mathrm{R}^{2}=\right.$ $\left.4-\mathrm{CH}_{3}\right)$ 的防效; 当测试浓度降至 $20 \mu \mathrm{g} / \mathrm{mL}$ 时, 化合物 10b $\left(\mathrm{R}^{2}=2-\mathrm{Br}\right)$ 和 10k $\left(\mathrm{R}^{2}=4-t-\mathrm{C}_{4} \mathrm{H}_{9}\right)$ 对东方粘虫仍具有 较好的杀虫效果, 致死率都为 $40 \%$, 明显高于其它化合 物的防效; 当 $\mathrm{R}^{1}$ 为 2 -氯噻唑-5-基时，化合物 10p 和 $10 \mathrm{q}$ 在 $100 \mu \mathrm{g} / \mathrm{mL}$ 浓度下对东方粘虫表现出 $100 \%$ 的杀虫活 性, 均高于对照药剂唑虫酰胺的防效 (50\%), 通过构效 关系分析可知，当苯环上引入氟、氯、溴吸电子基团时， 化合物 10I 100 对东方粘虫并未显示出杀虫效力, 当苯 环邻位引入甲氧基、三氟甲氧基给电子基团时，化合物 10p $\left(\mathrm{R}^{2}=2-\mathrm{OCH}_{3}\right)$ 和 10q $\left(\mathrm{R}^{2}=2-\mathrm{OCF}_{3}\right)$ 对东方粘虫的防 效要高于其它给电子基团化合物 10r 10t 的杀虫效果; 化合物 $10 p$ 和 $10 q$ 在浓度降到 $20 \mu \mathrm{g} / \mathrm{mL}$ 时，对东方粘 虫仍表现一定的杀虫效果, 致死率分别为 $20 \%$ 和 $30 \%$. 部分目标化合物在 $500 \mu \mathrm{g} / \mathrm{mL}$ 时对苜葆蚜呈现出较好的 杀虫活性，当 $\mathrm{R}^{1}$ 为 6-氯吡啶-3-基时，化合物 $10 \mathrm{~d}\left(\mathrm{R}^{2}=\right.$ $\mathrm{H}$ )对苜葆蚜具有 $60 \%$ 的杀虫效果; 当 $R^{1}$ 为 2 -氯噻唑-5基时，化合物 $10 \mathrm{~m}\left(\mathrm{R}^{2}=2-\mathrm{Cl}\right)$ 和 $100\left(\mathrm{R}^{2}=3-\mathrm{Br}\right)$ 对苜葆蚜 的杀虫活性分别为 $40 \%$ 和 $50 \%$. 另外, 目标化合物 10a 10t 在 $500 \mu \mathrm{g} / \mathrm{mL}$ 浓度下对朱砂叶螨均未表现出杀 虫效果, 这可能是由于药效团部分存在较大的基团, 导 致目标化合物与靶标结合受阻. 整体来看, 当 $R^{1}$ 为 6氯吡啶-3-基时, 化合物 $10 \mathrm{~b}\left(\mathrm{R}^{2}=2-\mathrm{Br}\right)$ 和 $10 \mathrm{k}\left(\mathrm{R}^{2}=\right.$ $\left.4-t-\mathrm{C}_{4} \mathrm{H}_{9}\right)$ 对东方粘虫的杀虫活性较为突出. 以上数据表 明, 将 $N$-吡啶基吡唑活性单元引入吡唑肜结构中, 得到 一些具有较好杀虫活性的目标化合物, 具有进一步优化 研究的价值.

\section{2 结论}

基于杀螨剂唑螨酯的分子结构, 将 $N$-吡啶基吡唑 结构引入到吡唑肪骨架中, 设计并制备了一系列结构新 颖的含 $N$-吡啶基吡唑单元的吡唑肜类化合物. 通过 ${ }^{1} \mathrm{H}$ 
表 2 目标化合物 10a 10t 的杀虫活性(死亡率/\%) ${ }^{a}$

Table 2 Insecticidal activities (mortality/\%) of the title compounds 10a $\sim 10 t$

\begin{tabular}{|c|c|c|c|c|c|c|}
\hline \multirow{2}{*}{ Compd. } & \multicolumn{3}{|c|}{ Oriental armyworm } & \multicolumn{2}{|c|}{ Aphis medicaginis } & \multirow{2}{*}{$\begin{array}{c}\text { Tetranychus cinnabarinus } \\
500 \mu \mathrm{g} \cdot \mathrm{mL}^{-1}\end{array}$} \\
\hline & $500 \mu \mathrm{g} \bullet \mathrm{mL}^{-1}$ & $100 \mu \mathrm{g} \cdot \mathrm{mL}^{-1}$ & $20 \mu \mathrm{g} \cdot \mathrm{mL}^{-1}$ & $500 \mu \mathrm{g} \cdot \mathrm{mL}^{-1}$ & $100 \mu \mathrm{g} \cdot \mathrm{mL}^{-1}$ & \\
\hline 10a & 100 & 0 & - & 0 & - & 0 \\
\hline $10 \mathrm{~b}$ & 100 & 100 & 40 & 0 & - & 0 \\
\hline 10c & 100 & 0 & - & 0 & - & 0 \\
\hline 10d & 100 & 100 & 0 & 60 & 0 & 0 \\
\hline $10 \mathrm{e}$ & 100 & 0 & - & 0 & - & 0 \\
\hline $10 f$ & 100 & 0 & - & 0 & - & 0 \\
\hline $10 \mathrm{~g}$ & 100 & 0 & - & 0 & - & 0 \\
\hline $10 \mathrm{~h}$ & 100 & 0 & - & 0 & - & 0 \\
\hline $10 \mathrm{i}$ & 100 & 0 & - & 0 & - & 0 \\
\hline $10 j$ & 100 & 0 & - & 0 & - & 0 \\
\hline $10 \mathrm{k}$ & 100 & 100 & 40 & 0 & - & 0 \\
\hline 101 & 100 & 0 & - & 0 & - & 0 \\
\hline $10 \mathrm{~m}$ & 100 & 0 & - & 40 & 0 & 0 \\
\hline $10 n$ & 100 & 0 & - & 0 & - & 0 \\
\hline 100 & 100 & 0 & - & 50 & 0 & 0 \\
\hline $10 p$ & 100 & 100 & 20 & 0 & - & 0 \\
\hline $10 q$ & 100 & 100 & 30 & 0 & - & 0 \\
\hline $10 \mathrm{r}$ & 100 & 0 & - & 0 & - & 0 \\
\hline $10 \mathrm{~s}$ & 100 & 0 & - & 0 & - & 0 \\
\hline $10 t$ & 100 & 0 & - & 0 & - & 0 \\
\hline Tolfenpyrad & 100 & 50 & 40 & - & - & - \\
\hline Imidacloprid & - & - & - & 100 & 100 & - \\
\hline Fenpyroximate & - & - & - & - & - & 100 \\
\hline
\end{tabular}

$\mathrm{NMR},{ }^{13} \mathrm{C} \mathrm{NMR}$ 和元素分析对新化合物进行了结构确 征, 并对其目标化合物进行了杀虫活性测试. 结果显示, 在 $500 \mu \mathrm{g} / \mathrm{mL}$ 浓度下, 所有目标化合物对东方粘虫都具 有 $100 \%$ 的杀虫效果, 与对照药剂唑虫酰胺的防效相当. 目标化合物 10b 和 10k 在 100 和 $20 \mu \mathrm{g} / \mathrm{mL}$ 浓度下对东 方粘虫仍表现出较好的杀虫活性. 目标化合物 $10 \mathrm{~d} 、 10 \mathrm{~m}$ 和 100 在 $500 \mu \mathrm{g} / \mathrm{mL}$ 浓度下对苜葆蚜呈现出 $40 \% \sim 60 \%$ 的杀虫活性. 从总的构效关系分析也可看出, $R^{1}$ 为 6-氯 吡啶-3-基时, 化合物 10b $\left(\mathrm{R}^{2}=2-\mathrm{Br}\right)$ 和 $10 \mathrm{k}\left(\mathrm{R}^{2}=4-t\right.$ $\mathrm{C}_{4} \mathrm{H}_{9}$ )对东方粘虫的杀虫效果较为突出, 可作为新型杀 虫先导结构, 为后续吡唑肜类衍生物的深入研究提供了 重要参考信息.

\section{3 实验部分}

\section{1 仪器与试剂}

核磁共振氢谱与碳谱数据采用 BRUKER $400 \mathrm{MHz}$ 核磁共振仪(TMS 为内标) 测定; 熔点使用 X-4 显微熔点 测定仪(温度计未经校正)测定; 元素分析使用 Yanaco-
CHN CORDER MT-3 自动元素分析仪测试. 实验所用的 试剂都为分析纯.

\section{2 化合物 1 3 和 $7 \sim 9$ 的合成}

化合物 1 3 的合成参照文献[32]，化合物 7 9 的 合成参照文献[33].

\section{3 化合物 4 的合成}

将中间体 3 (40 mmol)、2-氯-5-氯甲基吡定或 2-氯-5氯甲基噻唑 $(42 \mathrm{mmol}$ )、碳酸钾 $(44 \mathrm{mmol})$ 及 $80 \mathrm{~mL}$ 乙腈 加入反应瓶中, 加热回流反应 $8 \mathrm{~h}$. 然后将反应液冷却 至室温，经抽滤、浓缩得到中间体 4. 化合物 4 可直接用 于后面的反应. 为验证化合物 $\mathbf{4}$ 的结构，以取代基 $\mathrm{R}^{1}$ 为 6-氯吡啶-3-基的化合物为代表, 通过柱层析(以乙酸乙 酯/石油醚为洗脱剂, $V: V=1: 40)$ 分离得到. 浅黄色油 状物, 产率 $82 \%$. ${ }^{1} \mathrm{H}$ NMR $\left(\mathrm{CDCl}_{3}, 400 \mathrm{MHz}\right) \delta: 8.41(\mathrm{t}$, $J=2.20 \mathrm{~Hz}, 2 \mathrm{H}), 7.82 \sim 7.84(\mathrm{~m}, 1 \mathrm{H}), 7.72 \sim 7.75(\mathrm{~m}, 1 \mathrm{H})$, $7.52(\mathrm{~s}, 1 \mathrm{H}), 7.33 \sim 7.36(\mathrm{~m}, 1 \mathrm{H}), 7.28(\mathrm{~d}, J=4.02 \mathrm{~Hz}$, 1H), 5.29 (s, 2H), 4.19 (q, $J=7.20 \mathrm{~Hz}, 2 \mathrm{H}), 1.12$ (t, $J=$ 
7.20 Hz, 3H). Anal. calcd for $\mathrm{C}_{17} \mathrm{H}_{14} \mathrm{Cl}_{2} \mathrm{~N}_{4} \mathrm{O}_{3}$ : C 51.93, $\mathrm{H}$ 3.59, N 14.25; found C 51.76, H 3.73, N 14.38 .

\section{4 化合物 5 的合成}

将中间体 $4(25 \mathrm{mmol})$ 及 $30 \mathrm{~mL}$ 甲醇加入一反应瓶 中, 然后加热至 $45{ }^{\circ} \mathrm{C}$, 向其中缓慢滴加 $3 \mathrm{~mL} 20 \%$ 氢氧 化钠水溶液, 保持温度在 $45{ }^{\circ} \mathrm{C}$ 继续摚拌 $6 \mathrm{~h}$, 反应完成 后, 加入 $15 \mathrm{~mL}$ 水, 通过盐酸调节 $\mathrm{pH}$ 至 1 , 静置析出固 体, 经抽滤、烘干得中间体 $\mathbf{5}$, 不经纯化可直接用于后面 的反应.

\section{5 化合物 6 的合成}

将中间体 $5(5 \mathrm{mmol})$ 及 $20 \mathrm{~mL}$ 二氯甲烷加入一反应 瓶中, 室温搅拌下滴加草酰氯 $(15 \mathrm{mmol})$ 后, 再向其中滴 加 3 滴 $N, N$-二甲基甲酰胺(DMF), 然后继续室温搅拌 $3 \sim 4 \mathrm{~h}$, 反应结束后, 反应液经浓缩后得到中间体 6 , 未 经纯化直接用于后面的反应.

\section{6 目标化合物 10a 10t 的合成}

将中间体 $9(1 \mathrm{mmol}) 、 1 \mathrm{~mL}$ 三乙胺及 $20 \mathrm{~mL}$ 乙腈加 入一反应瓶中, 冰浴摚拌下, 向其中缓慢滴加 $1.5 \mathrm{mmol}$ 中间体 $\mathbf{6}$ 的乙腈 $(3 \mathrm{~mL})$ 溶液, 室温搅拌 $10 \sim 13 \mathrm{~h}$, 反应 完全结束后, 抽滤、浓缩后所得粗品通过甲醇重结晶, 得到目标产物 $10 \mathrm{a} \sim 10 \mathrm{t}$.

5-(4-氯苯氧基)-1,3-二甲基- $1 H$-吡唑-4-甲醛- $O$ - $\{1$ (3-氯吡啶-2-基)-3-[(6-氯吡啶-3-基)甲氧基]- $1 H$-吡唑-5甲酰基\}肜(10a): 白色固体, 产率 65\%. m.p. 146 $148{ }^{\circ} \mathrm{C} ;{ }^{1} \mathrm{H}$ NMR $\left(\mathrm{CDCl}_{3}, 400 \mathrm{MHz}\right) \delta: 8.45 \sim 8.46(\mathrm{~m}$, $2 \mathrm{H}), 7.94(\mathrm{~s}, 1 \mathrm{H}), 7.84 \sim 7.87(\mathrm{~m}, 1 \mathrm{H}), 7.74 \sim 7.77(\mathrm{~m}$, $1 \mathrm{H}), 7.31 \sim 7.38(\mathrm{~m}, 4 \mathrm{H}), 6.86(\mathrm{~d}, J=9.20 \mathrm{~Hz}, 2 \mathrm{H}), 6.47$ (s, 1H), $5.31(\mathrm{~s}, 2 \mathrm{H}), 3.60(\mathrm{~s}, 3 \mathrm{H}), 2.43(\mathrm{~s}, 3 \mathrm{H}) ;{ }^{13} \mathrm{C} \mathrm{NMR}$ $\left(\mathrm{CDCl}_{3}, 100 \mathrm{MHz}\right) \delta: 162.4,155.9,155.1,151.3,149.3$, 149.1, 148.7, 148.1, 147.0, 139.0, 138.6, 133.8, 131.0, 130.2, 129.6, 129.4, 125.5, 124.2, 116.7, 98.5, 97.6, 67.6, 34.5, 15.2. Anal. calcd for $\mathrm{C}_{27} \mathrm{H}_{20} \mathrm{Cl}_{3} \mathrm{~N}_{7} \mathrm{O}_{4}$ : C 52.91, H 3.29, N 16.00; found C 52.75, H 3.43, N 16.12 .

5-(2-溴苯氧基)-1,3-二甲基- $1 H$-吡唑-4-甲醛- $O$ - $\{1$ (3-氯吡啶-2-基)-3-[(6-氯吡啶-3-基)甲氧基]- $1 H$-吡唑-5甲酰基\}肜(10b): 白色固体, 产率 58\%. m.p. 159 $161{ }^{\circ} \mathrm{C} ;{ }^{1} \mathrm{H}$ NMR $\left(\mathrm{CDCl}_{3}, 400 \mathrm{MHz}\right) \delta: 8.44 \sim 8.47(\mathrm{~m}$, $2 \mathrm{H}), 7.67 \sim 7.85(\mathrm{~m}, 4 \mathrm{H}), 7.24 \sim 7.37(\mathrm{~m}, 3 \mathrm{H}), 7.06 \sim 7.10$ $(\mathrm{m}, 1 \mathrm{H}), 6.67 \sim 6.69(\mathrm{~m}, 1 \mathrm{H}), 6.48(\mathrm{~s}, 1 \mathrm{H}), 5.31(\mathrm{~s}, 2 \mathrm{H})$, $3.62(\mathrm{~s}, 3 \mathrm{H}), 2.44(\mathrm{~s}, 3 \mathrm{H}) ;{ }^{13} \mathrm{C} \mathrm{NMR}\left(\mathrm{CDCl}_{3}, 100 \mathrm{MHz}\right) \delta$ : $162.5,155.8,153.2,151.3,149.4,149.3,149.2,148.7$, $148.1,147.0,139.1,138.7,134.4,133.9,131.0,129.6$, 129.2, 125.7, 125.6, 124.2, 115.6, 111.4, 98.5, 97.7, 67.6, 34.5, 15.5. Anal. calcd for $\mathrm{C}_{27} \mathrm{H}_{20} \mathrm{BrCl}_{2} \mathrm{~N}_{7} \mathrm{O}_{4}$ : C 49.34, $\mathrm{H}$
3.07, N 14.92; found C 49.50, H 2.92, N 14.98 .

5-(3-溴苯氧基)- 1,3 -二甲基- $1 H$-吡唑-4-甲醛- $O$ - $\{1$ (3-氯吡啶-2-基)-3-[(6-氯吡啶-3-基)甲氧基]- $1 H$-吡唑-5甲酰基\}肜(10c)：白色固体，产率 60\%. m.p. 146 $147{ }^{\circ} \mathrm{C} ;{ }^{1} \mathrm{H}$ NMR $\left(\mathrm{CDCl}_{3}, 400 \mathrm{MHz}\right) \delta: 8.45 \sim 8.46(\mathrm{~m}$, $2 \mathrm{H}), 7.92(\mathrm{~s}, 1 \mathrm{H}), 7.74 \sim 7.86(\mathrm{~m}, 2 \mathrm{H}), 7.33 \sim 7.37(\mathrm{~m}$, $2 \mathrm{H}), 7.31(\mathrm{~d}, J=8.80 \mathrm{~Hz}, 1 \mathrm{H}), 7.09 \sim 7.25(\mathrm{~m}, 2 \mathrm{H}), 6.82 \sim$ $6.85(\mathrm{~m}, 1 \mathrm{H}), 6.48(\mathrm{~s}, 1 \mathrm{H}), 5.31(\mathrm{~s}, 2 \mathrm{H}), 3.60(\mathrm{~s}, 3 \mathrm{H}), 2.44$ $(\mathrm{s}, 3 \mathrm{H}) ;{ }^{13} \mathrm{C} \mathrm{NMR}\left(\mathrm{CDCl}_{3}, 100 \mathrm{MHz}\right) \delta$ : 162.5, 157.1, $155.8,151.3,149.3,149.1,148.9,148.6,148.2,147.0$, $139.0,138.6,133.8,131.4,131.0,129.6,127.5,125.2$, $124.2,123.5,118.9,113.9,98.6,97.6,67.6,34.5$, 15.3.Anal. calcd for $\mathrm{C}_{27} \mathrm{H}_{20} \mathrm{BrCl}_{2} \mathrm{~N}_{7} \mathrm{O}_{4}$ : C 49.34, H 3.07, N 14.92; found C 49.20, H 3.23, N 14.81.

5-苯氧基-1,3-二甲基- $1 H$-吡唑-4-甲醛- $O$ - $\{1$ - (3-氯吡 啶-2-基)-3-[(6-氯吡啶-3-基)甲氧基]- $1 H$-吡唑-5-甲酰基 $\}$ 肜(10d): 白色固体, 产率 62\%. m.p. 132 $134{ }^{\circ} \mathrm{C} ;{ }^{1} \mathrm{H}$ NMR $\left(\mathrm{CDCl}_{3}, 400 \mathrm{MHz}\right) \delta: 8.43 \sim 8.46(\mathrm{~m}, 2 \mathrm{H}), 7.89(\mathrm{~s}$, $1 \mathrm{H}), 7.74 \sim 7.83(\mathrm{~m}, 2 \mathrm{H}), 7.16 \sim 7.39(\mathrm{~m}, 5 \mathrm{H}), 6.92(\mathrm{~d}, J=$ $8.00 \mathrm{~Hz}, 1 \mathrm{H}), 6.46(\mathrm{~s}, 1 \mathrm{H}), 5.30$ (s, 2H), 3.60 (s, 3H), 2.44 $(\mathrm{s}, 3 \mathrm{H}) ;{ }^{13} \mathrm{C} \mathrm{NMR}\left(\mathrm{CDCl}_{3}, 100 \mathrm{MHz}\right) \delta: 162.5,156.7$, $155.9,151.3,149.9,149.4,149.1,149.1,148.0,147.0$, $139.0,138.6,133.9,131.0,130.3,129.6,125.5,124.3$, 124.2, 115.3, 98.5, 97.6, 67.6, 34.5, 15.5. Anal. calcd for $\mathrm{C}_{27} \mathrm{H}_{21} \mathrm{Cl}_{2} \mathrm{~N}_{7} \mathrm{O}_{4}$ : C 56.07, H 3.66, N 16.95; found C 56.18, $\mathrm{H} 3.83, \mathrm{~N} 16.81$.

5-(2-甲氧基苯氧基)-1,3-二甲基- $1 H$-吡唑-4-甲醛 - $O$ - \{1-(3-氯吡啶-2-基)-3-[(6-氯吡啶-3-基)甲氧基]- $1 H$-吡 唑-5-甲酰基\}肜(10e)：棕色固体，产率 57\%. m.p. 120 $122{ }^{\circ} \mathrm{C} ;{ }^{1} \mathrm{H}$ NMR $\left(\mathrm{CDCl}_{3}, 400 \mathrm{MHz}\right) \delta: 8.45 \sim 8.47(\mathrm{~m}$, $2 \mathrm{H}), 7.87(\mathrm{~s}, 1 \mathrm{H}), 7.75 \sim 7.86(\mathrm{~m}, 2 \mathrm{H}), 7.33 \sim 7.37(\mathrm{~m}$, $2 \mathrm{H}), 6.75 \sim 7.16(\mathrm{~m}, 4 \mathrm{H}), 6.43(\mathrm{~s}, 1 \mathrm{H}), 5.31(\mathrm{~s}, 2 \mathrm{H}), 3.91$ (s, 3H), 3.63 (s, 3H), $2.42(\mathrm{~s}, 3 \mathrm{H}) ;{ }^{13} \mathrm{C}$ NMR $\left(\mathrm{CDCl}_{3}, 100\right.$ MHz) $\delta: 162.4,155.9,151.3,150.8,149.4,149.2,149.2$, $149.1,147.8,147.0,145.4,139.1,138.7,133.9,131.0$, $129.6,125.5,125.4,124.2,121.1,116.5,113.0,97.7,97.5$, 67.6, 56.1, 34.4, 15.5. Anal. calcd for $\mathrm{C}_{28} \mathrm{H}_{23} \mathrm{Cl}_{2} \mathrm{~N}_{7} \mathrm{O}_{5}: \mathrm{C}$ 55.27, H 3.81, N 16.11; found C 55.40, H 3.70, N 16.27.

5-(4-甲氧基苯氧基)- 1,3 -二甲基- $1 \mathrm{H}$-吡唑-4-甲醛- $O$ \{1-(3-氯吡啶-2-基)-3-[(6-氯吡啶-3-基)甲氧基]-1H-吡唑 -5-甲酰基\}䏡(10f)：淡黄色固体，产率 55\%. m.p. 113 $115{ }^{\circ} \mathrm{C} ;{ }^{1} \mathrm{H}$ NMR $\left(\mathrm{CDCl}_{3}, 400 \mathrm{MHz}\right) \delta: 8.44 \sim 8.47(\mathrm{~m}$, $2 \mathrm{H}), 7.89(\mathrm{~s}, 1 \mathrm{H}), 7.75 \sim 7.85(\mathrm{~m}, 2 \mathrm{H}), 7.33 \sim 7.36(\mathrm{~m}$, 2H), 6.87 (s, 4H), $6.46(\mathrm{~s}, 1 \mathrm{H}), 5.31(\mathrm{~s}, 2 \mathrm{H}), 3.80(\mathrm{~s}, 3 \mathrm{H})$, $3.60(\mathrm{~s}, 3 \mathrm{H}), 2.43(\mathrm{~s}, 3 \mathrm{H}) ;{ }^{13} \mathrm{C} \mathrm{NMR}\left(\mathrm{CDCl}_{3}, 100 \mathrm{MHz}\right) \delta$ : 
$162.5,156.2,155.9,151.3,150.6,150.5,149.3,149.2$, $149.1,147.9,147.0,139.1,138.6,133.9,131.0,129.6$, 125.6, 124.2, 116.5, 115.2, 98.0, 97.6, 67.6, 55.8, 34.4, 15.5. Anal. calcd for $\mathrm{C}_{28} \mathrm{H}_{23} \mathrm{Cl}_{2} \mathrm{~N}_{7} \mathrm{O}_{5}$ : C 55.27, $\mathrm{H} \mathrm{3.81,} \mathrm{N}$ 16.11; found C 55.16, H 3.96, N 16.01.

5-(2-三氟甲氧基苯氧基)-1,3-二甲基- $1 H$-吡唑-4-甲 醛- $O$ - $\{1$-(3-氯吡啶-2-基)-3-[(6-氯吡啶-3-基)甲氧基]- $1 H$ 吡唑-5-甲酰基 $\}$ 肟(10g): 白色固体，产率 56\%. m.p. $150 \sim 152{ }^{\circ} \mathrm{C} ;{ }^{1} \mathrm{H}$ NMR $\left(\mathrm{CDCl}_{3}, 400 \mathrm{MHz}\right) \delta: 8.44 \sim 8.46$ $(\mathrm{m}, 2 \mathrm{H}), 7.74 \sim 7.86(\mathrm{~m}, 3 \mathrm{H}), 7.41 \mathrm{~d}, J=8.00 \mathrm{~Hz}, 1 \mathrm{H})$, $7.33 \sim 7.36(\mathrm{~m}, 2 \mathrm{H}), 7.18 \sim 7.26(\mathrm{~m}, 2 \mathrm{H}), 6.72 \sim 6.74(\mathrm{~m}$, $1 \mathrm{H}), 6.47$ (s, 1H), 5.30 (s, 2H), $3.61(\mathrm{~s}, 3 \mathrm{H}), 2.45$ (s, 3H); ${ }^{13} \mathrm{C} \mathrm{NMR}\left(\mathrm{CDCl}_{3}, 100 \mathrm{MHz}\right) \delta: 162.4,155.8,151.3,149.3$, $149.1,148.7,148.5,148.1,147.0,139.0,138.6,137.5$, 133.8, 131.0, 129.6, 128.6, 125.3, 121.9 (q, $J=257.20 \mathrm{~Hz}$ ), 115.6, 98.6, 97.7, 67.6, 34.4, 15.4. Anal. calcd for $\mathrm{C}_{28} \mathrm{H}_{20} \mathrm{Cl}_{2} \mathrm{~F}_{3} \mathrm{~N}_{7} \mathrm{O}_{5}$ : C 50.77, $\mathrm{H}$ 3.04, $\mathrm{N} \mathrm{14.80;} \mathrm{found} \mathrm{C}$ 50.94, H 2.90, N 14.67.

5-(4-三氟甲氧基苯氧基)-1,3-二甲基- $1 H$-吡唑-4-甲 醛- $O$ - $\{1$-(3-氯吡啶-2-基)-3-[(6-氯吡啶-3-基)甲氧基]- $1 H$ 吡唑-5-甲酰基 $\}$ 肜(10h): 白色固体，产率 63\%. m.p. $151 \sim 153{ }^{\circ} \mathrm{C} ;{ }^{1} \mathrm{H}$ NMR $\left(\mathrm{CDCl}_{3}, 400 \mathrm{MHz}\right) \delta: 8.45 \sim 8.46$ $(\mathrm{m}, 2 \mathrm{H}), 7.96(\mathrm{~s}, 1 \mathrm{H}), 7.74 \sim 7.86(\mathrm{~m}, 2 \mathrm{H}), 7.33 \sim 7.37(\mathrm{~m}$, 2H), 7.22 (d, $J=8.80 \mathrm{~Hz}, 2 \mathrm{H}), 6.94$ (d, $J=9.20 \mathrm{~Hz}, 2 \mathrm{H})$, $6.46(\mathrm{~s}, 1 \mathrm{H}), 5.30(\mathrm{~s}, 2 \mathrm{H}), 3.61(\mathrm{~s}, 3 \mathrm{H}), 2.43(\mathrm{~s}, 3 \mathrm{H}) ;{ }^{13} \mathrm{C}$ NMR $\left(\mathrm{CDCl}_{3}, 100 \mathrm{MHz}\right) \delta: 162.5,155.9,154.8,151.3$, $149.4,149.2,148.7,148.2,147.0,145.2,139.1,138.6$, 133.7, 131.0, 129.6, 125.5, 121.7 (q, $J=256.10 \mathrm{~Hz}$ ), 116.5, 98.5, 97.6, 67.6, 34.5, 15.2. Anal. calcd for $\mathrm{C}_{28} \mathrm{H}_{20} \mathrm{Cl}_{2}$ $\mathrm{F}_{3} \mathrm{~N}_{7} \mathrm{O}_{5}$ : C 50.77, H 3.04, N 14.80; found C 50.65, H 3.20, $\mathrm{N} 14.63$.

5-(3-甲基苯氧基)-1,3-二甲基- $1 H$-吡唑-4-甲醛- $O$ \{1-(3-氯吡啶-2-基)-3-[(6-氯吡啶-3-基)甲氧基]- $1 H$-吡唑 -5-甲酰基\}肪(10i): 白色固体，产率 59\%. m.p. 106 $108{ }^{\circ} \mathrm{C} ;{ }^{1} \mathrm{H}$ NMR $\left(\mathrm{CDCl}_{3}, 400 \mathrm{MHz}\right) \delta: 8.43 \sim 8.46(\mathrm{~m}$, $2 \mathrm{H}), 7.91(\mathrm{~s}, 1 \mathrm{H}), 7.74 \sim 7.83(\mathrm{~m}, 2 \mathrm{H}), 7.31 \sim 7.35(\mathrm{~m}$, 2H), $7.21 \sim 7.26(\mathrm{~m}, 1 \mathrm{H}), 6.98(\mathrm{~d}, J=7.60 \mathrm{~Hz}, 1 \mathrm{H}), 6.70$ $(\mathrm{d}, J=6.00 \mathrm{~Hz}, 2 \mathrm{H}), 6.46(\mathrm{~s}, 1 \mathrm{H}), 5.30$ (s, 2H), 3.59 (s, $3 \mathrm{H}), 2.45(\mathrm{~s}, 3 \mathrm{H}), 2.35(\mathrm{~s}, 3 \mathrm{H}) ;{ }^{13} \mathrm{C} \mathrm{NMR}\left(\mathrm{CDCl}_{3}, 100\right.$ $\mathrm{MHz}) \delta: 162.5,156.7,155.9,151.3,150.0,149.3,149.1$, $147.9,147.0,140.8,139.0,138.7,133.9,131.0,130.0$, $129.7,125.5,125.1,124.2,115.9,112.3,98.5,97.6,67.6$, 34.5, 21.5, 15.5. Anal. calcd for $\mathrm{C}_{28} \mathrm{H}_{23} \mathrm{Cl}_{2} \mathrm{~N}_{7} \mathrm{O}_{4}$ : C 56.77, H 3.91, N 16.55; found C 56.91, H 4.06, N 16.43.

5-(4-甲基苯氧基)-1,3-二甲基- $1 H$-吡唑-4-甲醛- $O$ -
\{1-(3-氯吡啶-2-基)-3-[(6-氯吡啶-3-基)甲氧基]- $1 H$-吡唑 -5-甲酰基\}肜(10j)：棕色固体，产率 65\%. m.p. 143 $145{ }^{\circ} \mathrm{C} ;{ }^{1} \mathrm{H}$ NMR $\left(\mathrm{CDCl}_{3}, 400 \mathrm{MHz}\right) \delta: 8.43 \sim 8.46(\mathrm{~m}$, 2H), $7.90(\mathrm{~s}, 1 \mathrm{H}), 7.74 \sim 7.84(\mathrm{~m}, 2 \mathrm{H}), 7.31 \sim 7.35(\mathrm{~m}$, $2 \mathrm{H}), 7.15(\mathrm{~d}, J=8.40 \mathrm{~Hz}, 2 \mathrm{H}), 6.80(\mathrm{~d}, J=8.80 \mathrm{~Hz}, 2 \mathrm{H})$, $6.46(\mathrm{~s}, 1 \mathrm{H}), 5.30(\mathrm{~s}, 2 \mathrm{H}), 3.59(\mathrm{~s}, 3 \mathrm{H}), 2.44(\mathrm{~s}, 3 \mathrm{H}), 2.34$ $(\mathrm{s}, 3 \mathrm{H}) ;{ }^{13} \mathrm{C} \mathrm{NMR}\left(\mathrm{CDCl}_{3}, 100 \mathrm{MHz}\right) \delta: 162.4,155.9$, $154.7,151.3,150.2,149.3,149.2,147.9,147.0,139.0$, $138.7,133.9,131.0,130.7,129.7,125.5,124.2,115.1$, 98.4, 97.6, 67.6, 34.4, 20.6, 15.5. Anal. calcd for $\mathrm{C}_{28} \mathrm{H}_{23}-$ $\mathrm{Cl}_{2} \mathrm{~N}_{7} \mathrm{O}_{4}$ : C 56.77, H 3.91, N 16.55; found C 56.60, H 3.82, $\mathrm{N} 16.70$.

5-(4-叔丁基苯氧基)-1,3-二甲基- $1 H$-吡唑-4-甲醛- $O$ \{1-(3-氯吡啶-2-基)-3-[(6-氯吡啶-3-基)甲氧基]- $1 H$-吡唑5-甲酰基\}肟(10k)：白色固体，产率 58\%. m.p. 135 $136{ }^{\circ} \mathrm{C} ;{ }^{1} \mathrm{H}$ NMR $\left(\mathrm{CDCl}_{3}, 400 \mathrm{MHz}\right) \delta: 8.43 \sim 8.46(\mathrm{~m}$, 2H), $7.90(\mathrm{~s}, 1 \mathrm{H}), 7.74 \sim 7.83(\mathrm{~m}, 2 \mathrm{H}), 7.31 \sim 7.42(\mathrm{~m}$, $4 \mathrm{H}), 6.83(\mathrm{~d}, J=8.40 \mathrm{~Hz}, 2 \mathrm{H}), 6.45(\mathrm{~s}, 1 \mathrm{H}), 5.30(\mathrm{~s}, 2 \mathrm{H})$, $3.60(\mathrm{~s}, 3 \mathrm{H}), 2.44(\mathrm{~s}, 3 \mathrm{H}), 1.32(\mathrm{~s}, 9 \mathrm{H}) ;{ }^{13} \mathrm{C} \mathrm{NMR}\left(\mathrm{CDCl}_{3}\right.$, $100 \mathrm{MHz}) \delta$ : 162.4, 155.9, 154.4, 151.2, 150.2, 149.3, $149.2,149.1,147.9,147.3,147.0,139.1,138.7,133.7$, $131.1,129.7,127.1,127.0,125.6,124.2,117.2,114.8$, 98.5, 97.6, 67.6, 34.4, 31.4, 15.4. Anal. calcd for $\mathrm{C}_{31} \mathrm{H}_{29^{-}}$ $\mathrm{Cl}_{2} \mathrm{~N}_{7} \mathrm{O}_{4}$ : C 58.68, H 4.61, N 15.45; found C 58.52, H 4.50, $\mathrm{N} 15.62$

5-(4-氟苯氧基)-1,3-二甲基- $1 H$-吡唑-4-甲醛- $O-\{1$ (3-氯吡啶-2-基)-3-[(2-氯噻唑-5-基)甲氧基]- $1 H$-吡唑-5甲酰基 \}肜(101): 褐色固体，产率 55\%. m.p. 120 $122{ }^{\circ} \mathrm{C} ;{ }^{1} \mathrm{H} \mathrm{NMR}\left(\mathrm{CDCl}_{3}, 400 \mathrm{MHz}\right) \delta: 8.47$ (d, $J=4.80$ $\mathrm{Hz}, 1 \mathrm{H}), 7.93$ (s, 1H), 7.87 (d, $J=8.00 \mathrm{~Hz}, 1 \mathrm{H}), 7.56$ (s, $1 \mathrm{H}), 7.36 \sim 7.39(\mathrm{~m}, 1 \mathrm{H}), 6.87 \sim 7.07(\mathrm{~m}, 4 \mathrm{H}), 6.43(\mathrm{~s}$, $1 \mathrm{H}), 5.41(\mathrm{~s}, 2 \mathrm{H}), 3.61(\mathrm{~s}, 3 \mathrm{H}), 2.42(\mathrm{~s}, 3 \mathrm{H}) ;{ }^{13} \mathrm{C} \mathrm{NMR}$ $\left(\mathrm{CDCl}_{3}, 100 \mathrm{MHz}\right) \delta: 161.9,159.1(\mathrm{~d}, J=242.10 \mathrm{~Hz})$, $155.9,153.3,152.5,149.8,149.1,148.9,148.1,147.0$, $141.4,139.1,135.2,134.0,129.6,125.6,116.9$ (d, $J=$ $19.50 \mathrm{~Hz}), 98.3,97.7,62.8,34.5$, 15.3.Anal. calcd for $\mathrm{C}_{25} \mathrm{H}_{18} \mathrm{Cl}_{2} \mathrm{FN}_{7} \mathrm{O}_{4} \mathrm{~S}$ : C 49.84, $\mathrm{H}$ 3.01, N 16.28; found $\mathrm{C}$ 49.75, H 3.14, N 16.12 .

5-(2-氯苯氧基)-1,3-二甲基- $1 H$-吡唑-4-甲醛- $O$ - $\{1$ (3-氯吡啶-2-基)-3-[(2-氯噻唑-5-基)甲氧基]- $1 H$-吡唑-5甲酰基 $\}$ 肜 $(10 \mathrm{~m})$ ：褐色固体，产率 52\%. m.p. 151 $153{ }^{\circ} \mathrm{C} ;{ }^{1} \mathrm{H}$ NMR $\left(\mathrm{CDCl}_{3}, 400 \mathrm{MHz}\right) \delta: 8.45$ (d, $J=4.00$ $\mathrm{Hz}, 1 \mathrm{H}), 7.85$ (d, J=6.00 Hz, 2H), 7.56 (s, 1H), 7.51 (d, $J=8.40 \mathrm{~Hz}, 1 \mathrm{H}), 7.35 \sim 7.39(\mathrm{~m}, 1 \mathrm{H}), 7.12 \sim 7.24(\mathrm{~m}, 2 \mathrm{H})$, $6.71(\mathrm{~d}, J=8.00 \mathrm{~Hz}, 1 \mathrm{H}), 6.45(\mathrm{~s}, 1 \mathrm{H}), 5.41(\mathrm{~s}, 2 \mathrm{H}), 3.62$ 
(s, 3H), $2.44(\mathrm{~s}, 3 \mathrm{H}) ;{ }^{13} \mathrm{C}$ NMR $\left(\mathrm{CDCl}_{3}, 100 \mathrm{MHz}\right) \delta$ : $161.9,155.8,153.3,152.1,149.4,149.1,148.7,148.1$, $147.0,141.4,139.1,135.2,134.0,131.3,129.7,128.4$, $125.6,125.3,122.8,115.8,98.4,97.8,62.8,34.5$, 15.4.Anal. calcd for $\mathrm{C}_{25} \mathrm{H}_{18} \mathrm{Cl}_{3} \mathrm{~N}_{7} \mathrm{O}_{4} \mathrm{~S}$ : C 48.52, H 2.93, N 15.84; found C 48.65, H 2.81, N 15.99 .

5-(3-氯苯氧基)- 1,3 -二甲基- $1 H$-吡唑-4-甲醛- $O$ - $\{1$ (3-氯吡啶-2-基)-3-[(2-氯噻唑-5-基)甲氧基]- $1 H$-吡唑-5甲酰基\}肜(10n): 白色固体，产率 58\%. m.p. 117 $118{ }^{\circ} \mathrm{C} ;{ }^{1} \mathrm{H}$ NMR $\left(\mathrm{CDCl}_{3}, 400 \mathrm{MHz}\right) \delta: 8.46 \sim 8.47$ (m, 1H), $7.93(\mathrm{~s}, 1 \mathrm{H}), 7.85 \sim 7.87(\mathrm{~m}, 1 \mathrm{H}), 7.56(\mathrm{~s}, 1 \mathrm{H}), 7.27 \sim$ $7.38(\mathrm{~m}, 2 \mathrm{H}), 6.78 \sim 7.17(\mathrm{~m}, 3 \mathrm{H}), 6.45(\mathrm{~s}, 1 \mathrm{H}), 5.41(\mathrm{~s}$, $2 \mathrm{H}), 3.61(\mathrm{~s}, 3 \mathrm{H}), 2.44(\mathrm{~s}, 3 \mathrm{H}) ;{ }^{13} \mathrm{C}$ NMR $\left(\mathrm{CDCl}_{3}, 100\right.$ MHz) $\delta: 161.9,151.1,155.8,153.3,149.1,149.0,148.7$, $148.2,147.0,141.4,139.1,135.8,135.2,134.0,131.1$, 129.7, 126.6, 124.6, 116.1, 113.5, 98.6, 97.7, 62.8, 34.5, 15.3. Anal. calcd for $\mathrm{C}_{25} \mathrm{H}_{18} \mathrm{Cl}_{3} \mathrm{~N}_{7} \mathrm{O}_{4} \mathrm{~S}$ : C 48.52, H 2.93, N 15.84; found C 48.38, H 3.08, N 15.71 .

5-(3-溴苯氧基)- 1,3 -二甲基- $1 \mathrm{H}$-吡唑-4-甲醛- $O$ \{1-(3-氯吡啶-2-基)-3-[(2-氯噻唑-5-基)甲氧基]- $1 H$-吡唑 -5-甲酰基\}肜(10o): 褐色固体, 产率 55\%. m.p. 118 $120{ }^{\circ} \mathrm{C} ;{ }^{1} \mathrm{H}$ NMR $\left(\mathrm{CDCl}_{3}, 400 \mathrm{MHz}\right) \delta: 8.46(\mathrm{~d}, J=3.60$ $\mathrm{Hz}, 1 \mathrm{H}), 7.93(\mathrm{~s}, 1 \mathrm{H}), 7.85 \sim 7.87(\mathrm{~m}, 1 \mathrm{H}), 7.56(\mathrm{~s}, 1 \mathrm{H})$, $7.35 \sim 7.38(\mathrm{~m}, 1 \mathrm{H}), 7.21 \sim 7.32(\mathrm{~m}, 2 \mathrm{H}), 7.10(\mathrm{~s}, 1 \mathrm{H})$, $6.82 \sim 6.85(\mathrm{~m}, 1 \mathrm{H}), 6.45(\mathrm{~s}, 1 \mathrm{H}), 5.41(\mathrm{~s}, 2 \mathrm{H}), 3.60(\mathrm{~s}$, $3 \mathrm{H}), 2.44(\mathrm{~s}, 3 \mathrm{H}) ;{ }^{13} \mathrm{C} \mathrm{NMR}\left(\mathrm{CDCl}_{3}, 100 \mathrm{MHz}\right) \delta: 161.9$, 157.0, 155.8, 153.3, 149.1, 149.0, 148.6, 148.2, 147.0, $141.4,139.1,135.2,134.0,131.4,129.6,127.5,125.6$, 123.5, 118.9, 113.9, 98.6, 97.7, 62.8, 34.5, 15.2. Anal. calcd for $\mathrm{C}_{25} \mathrm{H}_{18} \mathrm{BrCl}_{2} \mathrm{~N}_{7} \mathrm{O}_{4} \mathrm{~S}$ : C 45.27, H 2.74, N 14.78; found $\mathrm{C} 45.39, \mathrm{H} 2.99, \mathrm{~N} 14.61$.

5-(2-甲氧基苯氧基)-1,3-二甲基- $1 H$-吡夾-4-甲醛- $O$ \{1-(3-氯吡啶-2-基)-3-[(2-氯噻唑-5-基)甲氧基]- $1 H$-吡唑 -5-甲酰基\}肜(10p): 黄色固体, 产率 51\%. m.p. 123 $125{ }^{\circ} \mathrm{C} ;{ }^{1} \mathrm{H}$ NMR $\left(\mathrm{CDCl}_{3}, 400 \mathrm{MHz}\right) \delta: 8.39$ (d, $J=4.00$ $\mathrm{Hz}, 1 \mathrm{H}), 7.78 \sim 7.80(\mathrm{~m}, 2 \mathrm{H}), 7.50(\mathrm{~s}, 1 \mathrm{H}), 7.28 \sim 7.31(\mathrm{~m}$, $1 \mathrm{H}), 6.69 \sim 7.11(\mathrm{~m}, 4 \mathrm{H}), 6.33(\mathrm{~s}, 1 \mathrm{H}), 5.34(\mathrm{~s}, 2 \mathrm{H}), 3.84$ (s, 3H), 3.57 (s, 3H), 2.34 (s, 3H); ${ }^{13} \mathrm{C} \mathrm{NMR}\left(\mathrm{CDCl}_{3}, 100\right.$ MHz) $\delta: 160.8,154.9,152.3,149.8,148.2,148.0,146.8$, $146.0,144.3,140.4,138.1,134.2,133.1,128.6,126.8$, 124.6, 124.4, 120.1, 115.6, 112.0, 96.6, 96.5, 61.7, 55.1, 33.4, 14.5. Anal. calcd for $\mathrm{C}_{26} \mathrm{H}_{21} \mathrm{Cl}_{2} \mathrm{~N}_{7} \mathrm{O}_{5} \mathrm{~S}$ : C 50.82, $\mathrm{H}$ 3.44, N 15.96; found C 50.97, H 3.60, N 15.87.

5-(2-三氟甲氧基苯氧基)-1,3-二甲基- $1 H$-吡唑-4-甲 醛- $O$ - $\{1$-(3-氯吡啶-2-基)-3-[(2-氯噻唑-5-基)甲氧基]- $1 H$ - 吡唑-5-甲酰基\}肜(10q)：白色固体，产率 56\%. m.p. $129 \sim 131{ }^{\circ} \mathrm{C} ;{ }^{1} \mathrm{H}$ NMR $\left(\mathrm{CDCl}_{3}, 400 \mathrm{MHz}\right) \delta: 8.45 \sim 8.47$ $(\mathrm{m}, 1 \mathrm{H}), 7.85 \sim 7.87(\mathrm{~m}, 2 \mathrm{H}), 7.56(\mathrm{~s}, 1 \mathrm{H}), 7.35 \sim 7.42(\mathrm{~m}$, $2 \mathrm{H}), 7.18 \sim 7.26(\mathrm{~m}, 2 \mathrm{H}), 6.72 \sim 6.74(\mathrm{~m}, 1 \mathrm{H}), 6.44(\mathrm{~s}$, $1 \mathrm{H}), 5.41(\mathrm{~s}, 2 \mathrm{H}), 3.61(\mathrm{~s}, 3 \mathrm{H}), 2.45(\mathrm{~s}, 3 \mathrm{H}) ;{ }^{13} \mathrm{C} \mathrm{NMR}$ $\left(\mathrm{CDCl}_{3}, 100 \mathrm{MHz}\right) \delta$ : 161.9, 155.8, 153.8, 149.1, 148.7, $148.5,148.1,147.0,141.40,139.1,137.6,135.2,134.0$, 129.6, 128.6, 125.6, 121.9 (q, $J=257.10 \mathrm{~Hz}$ ), 115.6, 98.67, 97.8, 62.8, 34.2, 15.5. Anal. calcd for $\mathrm{C}_{26} \mathrm{H}_{18} \mathrm{Cl}_{2} \mathrm{~F}_{3} \mathrm{~N}_{7} \mathrm{O}_{5} \mathrm{~S}$ : C 46.72, H 2.71, N 14.67; found C 46.89, H 2.84, N 14.53.

5-(4-甲基苯氧基)-1,3-二甲基- $1 H$-吡唑-4-甲醛- $O$ \{1-(3-氯吡啶-2-基)-3-[(2-氯噻唑-5-基)甲氧基]- $1 H$-吡唑5-甲酰基\}肟(10r): 白色固体，产率 56\%. m.p. 132 $134{ }^{\circ} \mathrm{C} ;{ }^{1} \mathrm{H}$ NMR $\left(\mathrm{CDCl}_{3}, 400 \mathrm{MHz}\right) \delta: 8.45 \sim 8.46(\mathrm{~m}$, $1 \mathrm{H}), 7.90(\mathrm{~s}, 1 \mathrm{H}), 7.83 \sim 7.86(\mathrm{~m}, 1 \mathrm{H}), 7.56(\mathrm{~s}, 1 \mathrm{H}), 7.33 \sim$ 7.36 (m, 1H), 7.15 (d, $J=8.40 \mathrm{~Hz}, 2 \mathrm{H}), 6.80$ (d, $J=8.80$ $\mathrm{Hz}, 2 \mathrm{H}), 6.43$ (s, 1H), 5.41 (s, 2H), 3.59 (s, 3H), 2.44 (s, $3 \mathrm{H}), 2.34(\mathrm{~s}, 3 \mathrm{H}) ;{ }^{13} \mathrm{C} \mathrm{NMR}\left(\mathrm{CDCl}_{3}, 100 \mathrm{MHz}\right) \delta: 161.9$, $155.9,154.6,153.3,150.2,149.1,147.9,147.0,141.4$, $139.0,135.2,134.0,133.9,130.7,129.7,125.6,115.2$, 98.3, 97.6, 62.8, 34.4, 20.6, 15.4. Anal. calcd for $\mathrm{C}_{26} \mathrm{H}_{21}$ $\mathrm{Cl}_{2} \mathrm{~N}_{7} \mathrm{O}_{4} \mathrm{~S}$ : C 52.18, H 3.54, N 16.38; found C 52.34, H 3.41, N 16.52 .

5-(4-叔丁基苯氧基)- 1,3 -二甲基- $1 H$-吡唑-4-甲醛- $O$ \{1-(3-氯吡啶-2-基)-3-[(2-氯噻唑-5-基)甲氧基]- $1 H$-吡唑 -5-甲酰基\}肜(10s)：白色固体，产率 53\%. m.p. 131 $133{ }^{\circ} \mathrm{C} ;{ }^{1} \mathrm{H}$ NMR $\left(\mathrm{CDCl}_{3}, 400 \mathrm{MHz}\right) \delta: 8.44 \sim 8.46(\mathrm{~m}$, 1H), $7.90(\mathrm{~s}, 1 \mathrm{H}), 7.82 \sim 7.84(\mathrm{~m}, 1 \mathrm{H}), 7.56(\mathrm{~s}, 1 \mathrm{H}), 7.32 \sim$ $7.38(\mathrm{~m}, 3 \mathrm{H}), 6.83(\mathrm{~d}, J=8.80 \mathrm{~Hz}, 2 \mathrm{H}), 6.42(\mathrm{~s}, 1 \mathrm{H}), 5.41$ (s, 2H), 3.60 (s, 3H), 2.44 (s, 3H), 1.32 (s, 9H); ${ }^{13} \mathrm{C}$ NMR $\left(\mathrm{CDCl}_{3}, 100 \mathrm{MHz}\right) \delta: 161.9,155.9,154.4,153.3,150.2$, $149.2,149.1,147.9,147.3,147.0,141.4,139.0,135.2$, 134.0, 129.7, 127.1, 125.6, 114.8, 98.4, 97.6, 62.8, 34.5, 34.4, 31.2, 15.5. Anal. calcd for $\mathrm{C}_{29} \mathrm{H}_{27} \mathrm{Cl}_{2} \mathrm{~N}_{7} \mathrm{O}_{4} \mathrm{~S}$ : C 54.38, H 4.25, N 15.31; found C 54.27, H 4.39, N 15.46 .

5-(2,3-二甲基苯氧基)-1,3-二甲基- $1 H$-吡唑-4-甲醛$O$-\{1-(3-氯吡啶-2-基)-3-[(2-氯噻唑-5-基)甲氧基]- $1 H$-吡 唑-5-甲酰基\}肜(10t)：白色固体，产率 57\%. m.p. 141 $143{ }^{\circ} \mathrm{C} ;{ }^{1} \mathrm{H}$ NMR $\left(\mathrm{CDCl}_{3}, 400 \mathrm{MHz}\right) \delta: 8.45 \sim 8.46(\mathrm{~m}$, $1 \mathrm{H}), 7.83 \sim 7.85(\mathrm{~m}, 1 \mathrm{H}), 7.82(\mathrm{~s}, 1 \mathrm{H}), 7.56(\mathrm{~s}, 1 \mathrm{H}), 7.32 \sim$ $7.35(\mathrm{~m}, 1 \mathrm{H}), 6.97 \sim 7.03(\mathrm{~m}, 2 \mathrm{H}), 6.40(\mathrm{~d}, J=9.20 \mathrm{~Hz}$, $2 \mathrm{H}), 5.41(\mathrm{~s}, 2 \mathrm{H}), 3.58(\mathrm{~s}, 3 \mathrm{H}), 2.43(\mathrm{~s}, 3 \mathrm{H}), 2.36(\mathrm{~s}, 3 \mathrm{H})$, 2.31 (s, $3 \mathrm{H}) ;{ }^{13} \mathrm{C} \mathrm{NMR}\left(\mathrm{CDCl}_{3}, 100 \mathrm{MHz}\right) \delta: 161.9,155.9$, $154.7,153.3,150.6,149.2,149.1,147.9,147.0,141.4$, $139.5,139.1,135.3,134.1,129.7,126.5,125.8,125.6$, 
125.3, 111.4, 98.1, 97.6, 62.8, 34.3, 20.1, 15.5, 11.9. Anal. calcd for $\mathrm{C}_{27} \mathrm{H}_{23} \mathrm{Cl}_{2} \mathrm{~N}_{7} \mathrm{O}_{4} \mathrm{~S}$ : C 52.95, H 3.78, N 16.01; found C 52.82, H 3.94, N 16.15 .

\section{7 目标化合物 10a 10t 的生物活性测试}

对于目标物 $10 \mathrm{a} \sim 10 \mathrm{t}$, 用分析天平称取一定质量的 化合物原药, 用含吐温-80 乳化剂的 DMF 溶解配制为 $1.0 \%$ 母液, 接着用蒸馏水稀释, 每个重复处理 3 次, 并 设置空白对照. 试验所选昆虫分别为朱砂叶螨(Tetranychus cinnabarinus)、东方粘虫(Mythimna separata Walker) 和苜宿蚜(Aphis medicaginis). 对照药剂分别选择已商品 化的唑螨酯(纯度 99\%)、唑虫酰胺(纯度 $99 \%$ )和吡虫啉 (纯度 $99 \%$ ). 朱砂叶螨和苜宿蚜采用喷雾法 ${ }^{[34]}$ : 分别将 接有朱砂叶螨、苜宿蚜的蚕豆叶片于 Potter 喷雾塔下进 行喷雾处理, 然后将苜宿蚜放置在 $20{ }^{\circ} \mathrm{C}$ 的观察室内进 行培养, 朱砂叶螨放置在 $25{ }^{\circ} \mathrm{C}$ 的观察室内进行培养, $48 \mathrm{~h}$ 后观察实验的结果, 检查死活虫的数目, 进行数据 统计. 东方粘虫采用浸叶法 ${ }^{[35]}$ : 将玉米叶放置于配好的 药液中充分浸润, 自然阴干后, 再放于垫有滤纸的培养 皿中, 接东方粘虫 3 龄中期幼虫 10 头/血, 放在 $25{ }^{\circ} \mathrm{C}$ 的 观察室内进行培养, $48 \mathrm{~h}$ 后观察实验的结果, 检查死活 虫的数目, 进行数据统计.

\section{辅助材料(Supporting Information) 目标化合物 10a} $10 \mathrm{t}$ 的核磁共振氢谱和碳谱图谱. 这些材料可以免费从 本刊网站(http://sioc-journal.cn/)上下载.

\section{References}

[1] Guan, A. Y.; Qin, Y. K.; Wang, J. F.; Li, B. J. Fluorine Chem. 2013, $156,120$.

[2] Prakash, T. B.; Reddy, G. D.; Padmaja, A.; Padmavathi, V. Eur. J. Med. Chem. 2014, 82, 347.

[3] Pember, S. O.; Mejia, G. L.; Price, T. J.; Pasteris, R. J. Bioorg. Med. Chem. Lett. 2016, 26, 2965.

[4] Liu, X. H.; Zhao, W.; Shen, Z. H.; Xing, J. H.; Yuan, J.; Yang, G.; Xu, T. M.; Peng, W. L. Bioorg. Med. Chem. Lett. 2016, 26, 3626.

[5] Wang, B. L.; Shi, Y. X.; Zhang, S. J.; Ma, Y.; Wang, H. X.; Zhang, L. J.; Wei, W.; Liu, X. H.; Li, Y. H.; Li, Z. M. Eur. J. Med. Chem. 2016, 117,167

[6] Liu, X. H.; Zhao, W.; Shen, Z. H.; Xing, J. H.; Xu, T. M.; Peng, W. L. Eur. J. Med. Chem. 2017, 125, 881 .

[7] Shi, J. J.; Ren, G. H.; Wu, N. J.; Weng, J. Q.; Xu, T. M.; Liu, X. H.; Tan, C. X. Chin. Chem. Lett. 2017, 28, 1727.

[8] Mabkhot, Y. N.; Alharbi, M. M.; Al-Showiman, S. S.; Ghabbour, H. A.; Kheder, H. A.; Soliman, S. M.; Frey, W. Chem. Cent. J. 2018, $12,56$.

[9] Zhang, Y.; Zhan, Y. Z.; Ma, Y.; Hua, X. W.; Wei, W.; Zhang, X.; Song, H. B.; Li, Z. M.; Wang, B. L. Chin. Chem. Lett. 2018, 29 , 446

[10] Fu, Y.; Zhang, D.; Kang, T.; Guo, Y. Y.; Chen, W. G.; Gao, S.; Ye, F. Bioorg. Med. Chem. Lett. 2019, 29, 570.

[11] Zhong, L. K.; Jiang, T.; Zhang, F.; Fu, Q.; Liu, X. H.; Xu, T. M.;
Ding, C. R.; Chen, J.; Yuan, J.; Tan, C. X. Chin. J. Org. Chem. 2019, 39, 2655 (in Chinese).

(钟良坤, 江涛, 张帆, 付庆, 刘幸海, 许天明, 丁成荣，陈杰 袁静，谭成侠，有机化学, 2019, 39, 2655.)

[12] Wu, J.; Song, B. A.; Hu, D. Y.; Yue, M.; Yang, S. Pest Manage. Sci. 2012, 68, 801 .

[13] Wang, B. L.; Ma, Y.; Xiong, L. X.; Li, Z. M. Chin. J. Chem. 2012 30,815

[14] Yan, T.; Yu, S.; Liu, P.; Liu, Z.; Wang, B. L.; Xiong, L. X.; Li, Z. M. Chin. J. Chem. 2012, 30, 919.

[15] Wang, B. L.; Zhu, H. W.; Ma, Y.; Xiong, L. X.; Li, Y. Q.; Zhao, Y.; Zhang, J. F.; Chen, Y. W.; Zhou, S.; Li, Z. M. J. Agric. Food Chem. 2013, 61, 5483.

[16] Mao, M.; Li, Y.; Liu, Q.; Zhou, Y.; Zhang, X.; Xiong, L. X.; Li, Y. X.; Li, Z. M. Bioorg. Med. Chem. Lett. 2013, 23, 42.

[17] Wang, B. L.; Zhu, H. W.; Li, Z. M.; Wang, L. Z.; Zhang, X.; Xiong, L. X.; Song, H. B. Pest Manage. Sci. 2018, 74, 726.

[18] Hughes, K. A.; Lahm, G. P.; Selby, T. P.; Stevenson, T. M. WO 2004067528, 2004 [Chem. Abstr. 2004, 141, 190786].

[19] Wu, Z. B.; Zhou, X.; Ye, Y. Q.; Wang, P. Y.; Yang, S. Chin. Chem. Lett. 2017, 28, 121

[20] Liu, J. B.; Li, F. Y.; Li, Y. X.; Zhang, X. L.; Hua, X. W.; Xiong, L. X.; Li, Z. M. Pest Manage. Sci. 2019, 75, 1034.

[21] Zhang, Y.; Zhu, H. W.; Shang, J. F.; Wang, B. L.; Li, Z. M. Chin. J. Org. Chem. 2019, 39, 861 (in Chinese). (张燕，朱洪伟，尚俊峰，王宝雷，李正名，有机化学，2019，39, 861.)

[22] Shang, J. F.; Liu, Q. X.; Wang, B. L.; Li, Z. M. Chin. J. Org. Chem. 2019, 39, 1489 (in Chinese). (尚俊峰，刘巧霞，王宝雷，李正名，有机化学, 2019, 39, 1489.)

[23] Park, H. J.; Lee, K.; Park, S. J.; Ahn, B.; Lee, J. C.; Cho, H. Y.; Lee, K. I. Bioorg. Med. Chem. Lett. 2005, 15, 3307.

[24] Li, Y.; Zhang, H. Q.; Liu, J.; Yang, X. P.; Liu, Z. J. J. Agric. Food Chem. 2006, 54, 3636.

[25] Ouyang, G. P.; Cai, X. J.; Chen, Z.; Song, B. A.; Bhadury, P. S.; Yang, S.; Jin, L. H.; Xue, W.; Hu, D. Y.; Zeng, S. J. Agric. Food Chem. 2008, 56, 10160.

[26] Dai, H.; Chen, J.; Li, G.; Ge, S. S.; Shi, Y. J.; Fang, Y.; Ling, Y. Bioorg. Med. Chem. Lett. 2017, 27, 950.

[27] Swanson, M. B.; Ivancic, W. A.; Saxena, A. M.; Allton, J. D.; O'Brien, G. K.; Suzuki, T.; Nishizawa, H.; Nokata, M. J. Agric. Food Chem. 1995, 43, 513.

[28] Yang, H. Z.; Li, Q. Agrochemicals 2007, 46, 81 (in Chinese). (杨会芝，李庆，农药, 2007, 46, 81.)

[29] Fu, C. R.; Peng, J.; Ning, Y.; Liu, M.; Shan, P. C.; Liu, J.; Li, Y. Q.; Hu, F. Z.; Zhu, Y. Q.;Yang, H. Z.; Zou, X. M. Pest Manage. Sci. 2014, 70, 1207.

[30] Yang, Y. Z.; Lin, D. Y.; Fu, C. R.; Zou, X. M. Chin. J. Org. Chem. 2015, 35, 100 (in Chinese) (杨亚喆, 林大勇, 傅翠蓉, 邹小毛, 有机化学, 2015, 35, 100.)

[31] Shi, Y. J.; Wang, S. L.; He, H. B.; Li, Y.; Li, Y.; Fang, Y.; Dai, H. Chin. J. Org. Chem. 2015, 35, 1785 (in Chinese). (石玉军, 王森林, 何海兵, 李钰, 李阳, 方源, 戴红, 有机化学, 2015, 35, 1785.)

[32] Zhang, J. F.; Xu, J. Y.; Wang, B. L.; Li, Y. X.; Xiong, L. X.; Li, Y. Q.; Ma, Y.; Li, Z. M. J. Agric. Food Chem. 2012, 60, 7565.

[33] Dai, H.; Ge, S. S.; Li, G.; Chen, J.; Shi, Y. J.; Ye, L. Y.; Ling, Y. Bioorg. Med. Chem. Lett. 2016, 26, 4504.

[34] Dai, H.; Yao, W.; Fang, Y.; Sun, S. Y.; Shi, Y. J.; Chen, J.; Jiang, G. Q.; Shi, J. Molecules 2017, 22, 2000.

[35] Ding, C. R.; Pan, Y. Y.; Yin, X.; Tan, C. X.; Zhang, G. F. Chin. J. Org. Chem. 2019, 39, 836 (in Chinese).

(丁成荣，潘亚运，殷许，谭成侠，张国富，有机化学，2019，39, 836.)

$(\mathrm{Lu}, \mathrm{Y}$.) 